\title{
ARGUMENTELLIPSE IN DER "WEICHEN" NACHRICHT IM DEUTSCHEN UND IM SLOWENISCHEN
}

\section{EINFÜHRUNG}

Argumentellipsen werden in der sprachlichen Kommunikation ohne weiteres akzeptiert, obwohl sie allenfalls in gewissem Sinne wegen ihrer Elliptizität abweichend wirken. Im folgenden interessiert uns, wie die Argumentellipse als syntaktische Entität in den Text eingebettet wird und welche Funktionen sie ausübt. Unsere Aufmerksamkeit gilt hier der Textsorte "weiche" Nachricht, die als Abweichung des Grundmusters gilt. Um im Sprachenpaar Deutsch-Slowenisch die in dieser Textsorte realisierten Argumentellipsen vergleichen zu können, wurde die Homogenität in der Thematik angestrebt und die Texte aus dem gleichen Kommunikationsbereich gewählt. So wurden die Korpora zwei Zeitschriften, die sich mit der gleichen Sportart beschäftigen, entnommen: der deutschen Zeitschrift "Rotpunkt" und der slowenischen Zeitschrift "Grif".

\section{ZUM BEGRIFF DER ARGUMENTELLIPSE}

1.1 Der Begriff "Argumentellipse" wird vor dem Hintergrund der Valenztheorie definiert. Dieser Ellipsentyp stellt eine syntaktische Struktur dar, die das vom Valenzträger im Prädikat bestimmte syntaktische Minimum unterschreitet. Ein im tiefen Kasus angelegtes Argument, das die Proposition des Satzes mitbestimmt, wird nicht versprachlicht. ${ }^{1}$ Solche Sätze kommen im Deutschen und im Slowenischen vor. Die Definition geht daher von der Valenz- bzw. Argumentstruktur des Valenzträgers im Prädikat des Satzes aus. Die Argumentellipse betrifft beide Ergänzungsarten. Die Auslassung einer fakultativen Ergänzung ist hauptsächlich von pragmatischen Faktoren abhängig. ${ }^{2}$ Jedoch kann man feststellen, daß auch die obligatorischen Ergänzungen

1 Der Passivsatz mit der nicht verbalisierten Agensrolle wird nicht zu den Argumentellipsen gezählt, denn wegen der Passivbedeutung braucht diese Rolle grundsätzlich nicht realisiert zu werden.

2 Vgl. HELBIG 1992, 48: "Es ist zunächst syntaktisch feststellbar (und z. T. semantisch motivierbar), ob ein Aktant obligatorisch oder fakultativ ist. Ist er jedoch fakultativ, so ist es von der Kommunikationssituation abhängig (also eine Frage der pragmatischen Valenz), ob er realisiert wird 
ausgelassen werden, wenn diese Ergänzungen im jeweiligen textuellen bzw. situativen Kontext als bekannt vorausgesetzt werden können oder wenn dies die syntaktischen Faktoren ${ }^{3}$ ermöglichen. Solche Argumentellipsen kommen besonders häufig in der Umgangssprache vor, z.B.:

- Warum hast du mir nicht das Buch mitgebracht?

- _ _ Hab ich doch vorgestern per Post geschickt.

In der Antwort ist die Auslassung der obligatorischen Akkusativergänzung aufgrund der Tatsache möglich, daß sie die im Kontext bekannte Information darstellt.

In bestimmten Textsorten im Deutschen (Telegramm, Werbeanzeige, Inserat u.ä.) ist die Subjektellipse sogar prototypisch, obwohl das Subjekt zu den "obligatorischsten Ergänzungen" (vgl. HELBIG 1992) im Deutschen zählt. Z.B.:

- _ _ Suche Nachhilfe f. meinen Sohn (3. Graundschuljahr) in Mathematik. (Inserat)

Auch die Verwendung in einem bestimmten Kommunikationsbereich erlaubt die Auslassung von obligatorischen Aktanten. Der Satz Er sichert. ist wegen der Auslassung anormativ, jedoch wird er im Kommunikationsbereich "Bergsteigerwelt, Klettern" völlig akzeptiert. Durch das Vorwissen über den jeweiligen Kommunikationsbereich, vor dessen Hintergrund die Kommunikationspartner kommunizieren, sind die Informationen über die leeren Argumentstellen geliefert.

Die beschriebenen Auslassungen der obligatorischen Aktanten sind unter der Annahme möglich, daß die textlinguistisch-pragmatischen Faktoren die Auslassungen erlauben.

Das Subjekt wird im Slowenischen systembedingt ausgelassen und ist daher auch die am häufigsten ausgelassene fakultative Ergänzung. Dies entspricht im Slowenischen der Lesererwartung. Die pronominale Resyntaktifizierung des Subjekts im Text hat nicht nur textgrammatische, sondern auch pragmatische Funktionen. Da eine gründlichere Untersuchung der Subjektellipse im sprachlichen Kontext für das Slowenische noch aussteht, wird auf die Behandlung dieser Ergänzung in diesem Beitrag verzichtet.

1.2 Der erste Schwerpunkt meiner Untersuchung ist die Realisierung der Argumentellipse in schriftlichen Texten. Argumentellipsen werden als satzwertige Textelemente verstanden, deren Satzbedeutung durch das Heranziehen des Kontextes sinnfällig wird. ${ }^{4}$ Die Argumentellipse wird transphrastisch, aus textlinguistischpragmatischer Sicht betrachtet.

oder nicht."

3 Auch syntaktische Faktoren erlauben bei einigen Verben die Auslassung sonst obligatorischer Aktanten. Ein Beispiel dafür ist das Verb geben, dessen Argumente im Verb-Zweitsatz realisiert werden müssen, im Imperativsatz jedoch nicht: Gib!, Gib das Buch! Dies ist möglich unter der Annahme, daß das Argument im schriftlichen oder situativen Kontext verankert ist.

4 Die s.g. konventionalisierte Ellipse wie z.B. das in der Fachliteratur meist zitierte Beispiel Die Henne legt. wird nicht in die Untersuchung einbezogen, da die leere Argumentstelle nicht aus dem 
Die Einbettung in den Kontext wird durch die Distinktion definit/indefinit ${ }^{5}$ plausibel gemacht. Sehen wird uns ein Beispiel wie Leo liest. oder im Slowenischen Peter bere. an. Das nicht-syntaktifizierte Argument (Akkusativergänzung) bleibt implizit, es ist auf der semantischen Ebene vorhanden. Die nicht besetzte Argumentstelle wird durch die Übersetzung in die Proposition deutlich LES(LEO,X2) bzw. BER(PETER, X2). Durch kontextabhängige Interpretationsregeln kann die leere Argumentstelle $\mathrm{X} 2$ verschiedene Interpretationen erhalten, denn sie kann auf einen indefiniten oder auf einen definiten Argumentausdruck zurückgeführt werden. Es geht nicht um die semantische, sondern um die referentielle Mehrdeutigkeit. Wenn die leere Argumentstelle keinen Bezug auf den Kontext nimmt, kann die Argumentellipse ohne Kontext isoliert verstanden werden, sie hat einen indefiniten Referenzbezug. In diesem Fall kann die leere Argumentstelle durch das indefinite Pronomen jemand oder etwas ergänzt werden. Es handelt sich um den indefiniten Referenzbezug, die Argumentellipse ist indefinit.

Wenn sich die leere Argumentstelle im Satz auf ein im Kontext verankertes Element bezieht, ist ihr Referenzbezug definit. Es wird etwas ganz Bestimmtes unter der Zweitstelle von lesen/brati verstanden. Dies wird durch die Kontextinformation deutlich. Im Beispiel Leo hat sich heute morgen eine Zeitung gekauft. Jetzt liest er. wirkt die leere Argumentstelle rückwärtsweisend, sie ist referentiell identisch mit dem Textelement eine Zeitung des vorangegangenen Satzes. Somit dient sie zur Referenzkontinuität.

1.3 In bezug auf die referentiellen Auswirkungen entwickelte Sigrid DENTLER in ihrem Werk "Verb und Ellipse im heutigen Deutsch" (1990) Kontexttypen zur Argumentellipse. Die wichtigsten Kriterien für ihre Kontexttypologie sind (zusammenfassend): Der Referenzbezug der leeren Argumentstelle im Text ist definit oder indefinit, er nimmt an dem "Szenariowisssen"6 im Text teil, das koreferentielle Textelement mit der leeren Stelle ist im Text explizit oder implizit fokussiert und kann im Nah- oder Fernverweis stehen, der Referenzbezug ist relevant für die Textkohärenz.

Daraus geht hervor, daß die Argumentellipse ein effektives Mittel zur Bildung der Textkohärenz ist. Sie entsteht im Textgestaltungsprozeß nach der Feststellung ihrer Angemessenheit, ${ }^{7}$ ist einerseits eines der Bestandteile des Textes ${ }^{8}$ und andererseits das Resultat des adressatenbezogenen kommunikativen Handelns. Welche Rolle spielt sie in der "weichen" Nachricht?

Kontext ergänzt wird. Vom Sprecher wird sie immer auf gleiche Weise aktualisiert.

5

Vgl. SHOPEN 1972.

6 Nach DENTLERS Anmerkung wird "Szenariowissen" im Sinne von Sanford/Garrod (1981, zit. nach DENTLER 1990) verstanden: Es geht um eine Subsumierung vom Frame-, Scriptwissen wie auch enzyklopädischen Kenntnissen.

7 Vgl. DeBEAUGRANDE/DRESSLER 1981, 74.

8 Der Text wird hier sowohl im zeichenorientierten, sprachsystematischen Ansatz als auch im handlungstheoretischen, pragmatischen Ansatz verstanden. Beide Ansätze können komplementär verstanden werden, da der Text als organisierte Folge von Sätzen unter konkreten situativen Bedingungen entsteht und das Resultat einer sprachlich-kommunikativen Handlung ist. 


\section{ZUM BEGRIFF DER "WEICHEN" NACHRICHT}

2.1 Zuerst einige Erläuterungen zum Begriff "weiche" Nachricht. Die Textsorte "Pressenachricht" gehört dem usuellen Typus an. ${ }^{9}$ Als Hauptmerkmale werden häufig folgende Eigenschaften der Nachricht aufgezählt: informierend, aktuell, interessant, als Gegenstand Sachverhalte aus den Sparten Politik, Wirtschaft, Kriminalität u. ä. jedoch ohne ein festgelegtes Thema; monologisch, sachbetont, kurz, ohne persönliche Wertung, mit relativ schematischem Aufbauprinzip; Klarheit, Genauigkeit. Oft wird zur Definition der Nachricht auch das didaktische Prinzip der Nachricht herangezogen, das besagt, daß die Nachricht auf 5 W-Fragen antwortet, nämlich wer, wann, wo, was, wie oder warum, da diese Antworten den Einbezug der wichtigsten Angaben auf kürzeste Weise sichern. Die "weiche" Nachricht entsteht durch eine abweichende Realisierung des Grundmusters. Sie beinhaltet die Antworten auf die W-Fragen, jedoch erhält diese Textsorte außer der Textfunktion "Informieren" noch die Textfunktion "Unterhalten". Deswegen wird sie sprachlich besonders abwechslungsreich realisiert. So auch LÜGER (1995, 103): "Liegt beim Typ der harten Nachricht ein relativ schematisches Aufbauprinzip mit sachlich wirkendem Sprachstil vor, so zeichnen sich weiche Nachrichten gerade durch variationsreiche Textgestaltung und lesewerbende Informationspräsentation aus. Gegenstand sind nicht so sehr Sachverhalte aus den Sparten Politik oder Wirtschaft, sondern Skandale, Verbrechen, Naturkatastrophen, Unglücksfälle; oder man berichtet über Einzelheiten aus dem Leben bekannter Persönlichkeiten usw./.../Die Bedeutung der Themen ergibt sich vor allem aus der Möglichkeit sensationeller Aufmachung, aus dem Unterhaltungswert für das Leserpublikum." Die weichen Nachrichten werden von der Lesererwartung mitbestimmt und finden sich häufig bei dem nichttagesaktuellen Umfeld. Besonders typisch sind sie für spezielle Kommunikationsbereiche, in denen eine bestimmte Lesererwartung eine große Rolle spielt.

2.2 Die "weichen" Nachrichten sind der deutschen Kletterzeitschrift "Rotpunkt" und einer vergleichbaren slowenischen Zeitschrift, dem Sportmagazin "Grif", entnommen worden. ${ }^{10}$ Es handelt sich um spezialisierte Zeitschriften, die Informationen über die Sportdisziplin "Klettern" aus dem Heimatland und der ganzen Welt bringen. In bezug auf das Trägermedium sind in beiden Zeitschriften die meisten Textsorten der Presse vertreten, die jedoch alle auf die Kletterthematik bezogen sind. Beide Zeitschriften richten sich an einen bestimmten Adressaten - das sind Hochleistungskletterer, Freizeitsportler, Alpinisten und auch alle anderen, die an diesem Sport interessiert sind. Demzufolge sind die Leser eine Interessengruppe, die die Zeitschrift wegen ihres Hobbys kauft und liest. Aus diesen sozialsituativen ${ }^{11}$

9 Vgl. HEUSINGER 1995b, 15.

10 Sie erscheinen zweimonatlich, "Rotpunkt" seit 1986 und "Grif" seit 1995.

11 Unter der sozialen Situation werden hier alle diejenigen Faktoren verstanden, die die Informationen über die Kommunikationspartner angeben, wie z.B. der Grad der Vertraulichkeit der 
Faktoren läßt sich schlußfolgern, daß die Kommunikationspartner über ähnliches Vorwissen verfügen, d. h. Fachwissen (zur speziellen Sportdisziplin) und Szenariowissen. Der Sender erwartet, daß der Leser bei der Lektüre sein Vorwissen aktivieren wird. ${ }^{12}$

Den Nachrichten in "Rotpunkt" und "Grif" liegen in bezug auf das Spezialfach Sachverhalte wie Ereignisse einzelner Klettersportler, Alpinisten und Expeditionen, Eröffnungen oder Sperrungen der Klettergebiete u.ä. zugrunde. Da einerseits die Thematik sehr einheitlich, sogar eng auf eine Sportart bezogen ist, und da andererseits die Leser das Klettern als Freizeitbeschäftigung ansehen, sollen die Informationen sprachlich variationsreich vermittelt werden. Aus diesen Gründen versucht sowohl "Rotpunkt" wie auch "Grif" eine lockere, attraktive und gleichzeitig eine spannende Atmosphäre für den Leser zu schaffen. Der Leser soll nicht nur informiert werden, er soll auch Vergnügen an der Lektüre haben. Die Sprachverwendung ist der Lesererwartung angepaßt. So sind auch die Texteröffnung und der Textschluß abweichend gestaltet, auch die Abfolge von Informationen ist anders als bei den neutral gestalteten Nachrichten. Wie gezeigt wird, werden die Nachrichten in der deutschen wie auch in der slowenischen Kletterzeitschrift weitgehend als "weiche" Nachricht realisiert. Sehen wir uns einige Beispiele an:

\section{Überschrift: Schwindelnde Höhen}

Texteröffnung: In solchen scheint sich der Franzose Fred Rouhling zu bewegen, zumindest wenn man seinem Statement Glauben schenken darf, er habe mit seiner neuesten Schöpfung "Akira" gerade der Welt erste $9 b$ eröffnet. Zugetragen hat sich das Unglaubliche nahe Vilhonneur (Charente),... (aus: Rotpunkt 6/95)

Überschrift: Sova v Ospu in na Gorenjskem

Texteröffnung: $\mathrm{Na}$ veliko žalost ljubiteljev ptičev ter ostale pernate divjadi in sovražnikov plezalcev v Ospu nismo videli na primer Velike uharice, temveč Mateja Sovo, kako je 19. junija opravil s smerjo Highlander... ${ }^{13}$ (aus: Grif 2/7)

Die Aufmerksamkeit des Lesers wird mit verschiedenen sprachlichen Mitteln erregt, wie z. B. durch Nutzung rhetorischer Figuren, Wortspiele und Idiome (Glauben schenken; Sova - dt. die Eule, gleichzeitig auch ein slowenischer Familienname),

Kommunikationspartner, ihr sozialer Status, ihre Zugehörigkeit zur bestimmten Kultur, zum bestimmten Milieu; ihre Interessen, Wünsche, Bedürfnisse, weltanschauliche/religiöse Bindung usw.

12 Besonders charakteristisch für die sozialen Verhältnisse zwischen Kommunikationspartnern in "Rotpunkt" ist beispielsweise die Anredeform. Der Leser wird nicht mit "Sie", sondern mit der 2. Person Plural "Ihr" angesprochen, d.h. als Teil einer wir-Gruppe, mit der er sich identifizieren kann. Damit gelingt der Zeitschrift eine Atmosphäre zu schaffen, die ins Private rückt, die Distanz zwischen dem Sender und dem Adressaten wird kürzer.

13 Deutsche Übersetzung: Zum Leidwesen der Freunde des Federwildes und Kletterfeinde kam in Osp kein Uhu zum Vorschein, sondern Matej Sova, der am 19. Juni die Route Highlander klettern konnte... 
Verwendung von Anglizismen (Statement), Aufnahme origineller, im Kontext überraschend wirkender Informationen (die Uhus in Osp sind bedroht), Einführung einer nicht ernsten Modalität oder Ironie-Signale (Zugetragen hat sich das Unglaubliche...) usw. Zahlreiche sprachliche Mittel haben eine starke lesewerbende Funktion, die Textfunktion der "weichen" Nachricht ist nicht nur "Informieren", sondern auch "Unterhalten".

\section{3 "WEICHE" NACHRICHT UND ARGUMENTELLIPSE}

3.1 Kehren wir zur Argumentellipse zurück. In 17 Ausgaben von "Rotpunkt" wurden 71 Nachrichten mit 125 Argumentellipsen gefunden. In 10 Ausgaben von "Grif" wurden 30 Nachrichten mit 46 Argumentellipsen gefunden.

Bei der Identifizierung der Argumentellipsen stützte ich mich auf die Lexikoneintragungen in den Valenzwörterbüchern der deutschen Sprache. ${ }^{14}$ Dabei stieß ich auf manche Probleme: Diese Valenzwörterbücher umfassen nicht alle Verben, die in meiner Untersuchung zur Analyse herangezogen wurden. Andererseits unterscheiden sich die Lexikoneintragungen zum gleichen Verb untereinander wesentlich. ${ }^{15}$ Für die slowenischen Verben verwendete ich das Wörterbuch der slowenischen Schriftsprache, ${ }^{16}$ da fürs Slowenische noch kein Valenzwörterbuch entwickelt worden ist.

3.2 Die Tabelle 1 zeigt, daß in den untersuchten Texten nicht nur fakultative, sondern auch obligatorische Ergänzungen ausgelassen werden.

Tabelle 1: Ausgelassene Ergänzungen in den Argumentellipsen

\begin{tabular}{|l|l|l|l|l|}
\hline ausgelassene Ergänzungen & \multicolumn{2}{|c|}{ Rotpunkt-Nachrichten } & \multicolumn{2}{c|}{ Grif-Nachrichten } \\
\hline & Zahl & $\%$ & Zahl & $\%$ \\
\hline obligatorisch & 19 & 15 & 9 & 19 \\
\hline fakultativ & 106 & 85 & 38 & 82 \\
\hline Ergän. insgesamt & 125 & 100 & 47 & 100 \\
\hline
\end{tabular}

Jedoch ist die kategoriale Charakterisierung der ausgelassenen obligatorischen Ergänzungen in slowenischen und deutschen Belegen unterschiedlich. In den deutschen Argumentellipsen werden Subjekte, Dativergänzungen, Präpositionalphrasen und Akkusativergänzungen ausgelassen. Am häufigsten werden die obligatorischen Akkusativergänzungen ausgelassen (z.B. Klettergärten und

14 HELBIG/SCHENKEL (1991), GERLING/ORTHEN (1979) und ENGEL/SCHUMACHER (1976).

15 Wenn wir die Lexikoneintragungen zu den einzelnen Verben untereinander vergleichen, kann man sehen, daß sie sich untereinander wesentlich unterscheiden, und zwar in Hinblick auf die Zahl der Aktanten wie auch in Hinblick auf ihre Obligatorität. Siehe z.B. Verben sich bewerben, sitzen u.a. 
Hallenwände sind out. Macht schließlich jeder; All jene, die bereits vorbestellt haben, bitten wir noch um ein wenig Geduld.), da der Referenzbezug im Kontext stark verankert ist. Die Auslassungen der Subjekte im Deutschen sind umgangssprachlich markiert (z.B. Mit Bild, versteht sich.). Ausgelassen wurden noch einige obligatorische Präpositionalergänzungen (z. B. Ungewöhnlich war auch die eigentliche Arbeit an der Route, da Alex am Ausstieg begann und sich nach unten durcharbeitete.) und Dativergänzungen (z.B. Vorgeworfen wurde die französiche Idee, in einigen Artikeln ein gemeinsames Layout anzustreben.). Diese Auslassungen sind auf einen Trend in der Umgangssprache zurückzuführen.

Im Slowenischen kommen zwei Verben vor, deren obligatorische Aktanten ausgelassen werden: Es handelt sich um das zweiwertige Verb nadaljevati (dt. fortsetzen), das einwertig gebraucht wird (z.B. ...in čez dve uri stal na Severnem sedlu ter od tam naprej nadaljeval sam.) und dessen Ergänzung im Kontext stark verankert ist, und um das dreiwertige opremiti (dt. versehen, ausstatten, z.B. ...Borut Kavzar pa je $v$ Kotečniku opremil novo smer, jo poimenoval Specialist za življenje in jo po uspešnem vzponu ocenil z $8 a+$.), das zweiwertig gebraucht wird. Der erfahrene Leser dieser Zeitschrift kann das ausgelassene Argument im Kommunikationsbereich "Klettern" mit seinem speziellen Wissen leicht ergänzen.

Tabelle 2: Ausgelassene fakultative Ergänzungen nach ihrer kategorialen Charakterisierung

\begin{tabular}{|l|l|l|l|l|}
\hline fakult. Erg. & \multicolumn{2}{|l|}{ Rotpunkt-Nachrichten } & \multicolumn{2}{l|}{ Grif-Nachrichten } \\
\hline & Zahl & $\%$ & Zahl & $\%$ \\
\hline DatNP & 55 & 52 & 15 & 39 \\
\hline AkkNP & 10 & 9 & 1 & 3 \\
\hline PP & 41 & 39 & 22 & 58 \\
\hline insgesamt & 106 & 100 & 38 & 100 \\
\hline
\end{tabular}

Die kategoriale Charakterisierung der ausgelassenen fakultativen Ergänzungen in den deutschen Belegen unterscheidet sich wenig von der Charakterisierung der Auslassungen im Slowenischen. In den Rotpunkt-Nachrichten wäre die Hälfte der leeren Argumentstellen mit den Dativergänzungen zu besetzen, die nicht realisierte semantische Rolle ist der Rezipient. Meistens geht es um indirekte Objekte, ein dreiwertiges Verb wird zweiwertig gebraucht. (Es werden Dativergänzungen bei dreiwertigen Verben ausgelassen wie bieten, erklären, versprechen, zeigen, präsentieren, liefern, verraten, verbieten.) Ähnlich gebraucht werden auch dreiwertige slowenische Verben (z.B. postreči, dokazati, podeliti, zlagati se, ponujati, pomagati, odgovoriti, obljubljati, nuditi, predlagati, dodati, poslati, ponujati, povedati).

3.3 Der definite/indefinite Referenzbezug der leeren Argumentstelle bei ausgelassenen fakultativen Ergänzungen: Die hohe Anzahl der definiten Argumentellipsen in deutschen $(72 \%)$ und slowenischen $(76 \%)$ weichen Nachrichten besagt, 
daß die unbesetzten Argumentstellen in hohem Maße zur Referenzkontinuität beitragen. Die Interpretation dieser leeren Stellen ist demnach stark vom schriftlichen Kontext abhängig. Wie erwartet, sind die meisten leeren definiten Argumentstellen in den Nachrichten anaphorisch, und zwar im deutschen wie auch im slowenischen Sprachmaterial (92\%).

Jedoch sind seltene leere Positionen auch vorwärtsweisend (wie z.B. Nicht anders ist es zu erklären, daß mitten im Sommer gleich drei herausragende Rotpunktbegehungen im alpinen Fels gelangen. Mitte August konnte Thomas Huber ein langjähriges Projekt an der Feuerhorn-Nordwand vollenden, das sich seit 1985 wie ein roter Faden durch die Kletterwelt des Chiemgauers zog...) Die ausgelassene Dativergänzung koreferiert mit dem Textelement Thomas Huber aus dem nachgestellten Satz.

3.4 Wie werden die definiten Argumentellipsen in den Kontext eingebettet?

a) Die leere Stelle bezieht sich auf ein Textelement, das explizit auf der Textoberfläche und im Nahverweis vorkommt. ${ }^{17}$ Die meisten definiten Argumentellipsen kommen in diesem Kontext vor (41\% von den deutschen, $55 \%$ von den slowenischen Argumentellipsen).

Beispiel aus der Rotpunkt-Nachricht:

Lynn setzt alles auf eine Karte, und dieses mal gelingt es

Im zweiten Teil der Koordination wurde die fakultative Dativergänzung bzw. die semantische Rolle des Rezipienten nicht versprachlicht. Es ist klar, daß hier das Indefinitpronomen jemand, das die Indefinitheit des Bezugs angeben könnte, nicht antrifft, denn mit der Frage Wem gelingt es nicht? wird nach einer bestimmten Person gefragt, die im vorangehenden Satz schon erwähnt wurde: Lynn. Der Referenzbezug der leeren Position ist also anaphorisch verankert, er wird explizit fokussiert. Da das ausgelassene Argument vom Leser implizit verstanden wird, unterstiutzt die Argumentellipse hier die Referenzkontinuität im Text und lenkt die Aufmerksamkeit des Lesers auf die Bedeutung des im Verb ausgedrückten Ereignisses.

Beispiel aus der Grif-Nachricht:

Dušan Polenik in Tedej Golob sta se 5. oktorbra zjutraj komaj rešila iz podirajočega šotora, ki sta ga postavila pod južno steno Južnega Nilgirija (6838).

Ko sta se čez nekaj dni vrnila, ni bilo šotora in opreme, ki sta jo za vzpon čez 1800 visoko steno znosila vanj, nikjer več.

In diesem Kontext korreliert die leere Argumentstelle beim Verb vrniti se (dt. zurückkehren) mit dem explizit fokussierten Textelement, hier eine Nominalphrase podirajoči šotor (+Relativsatz), die leere Position wirkt anaphorisch.

Im Rahmen unserer Materialbreite kann man feststellen, daß dieser Kontexttyp in den slowenischen wie auch in deutschen leichten Nachrichten am häufigsten

17 Nach DENTLER (1990) geht es um den Kontexttyp A. 
vorkommt. Dies läßt sich mit den Textsortenkonventionen in Verbindung bringen. Wenn das Bezugselement auf der Textoberfläche vorkommt, kann der Leser schneller die leere Stelle mit dem koreferentiellen Element verbinden. Die Information aus dem Text wird somit für den Leser plausibler. Gleichzeitg trägt dieser Kontexttyp zur stärkeren Kohärenz zwischen den Sätzen einer kürzeren Textsequenz bei. Die Satzkette kann durch referentielle Beziehungen zwischen der Argumentellipse und dem Bezugselement im Text kohärent gebildet werden.

b) Die leere Stelle bezieht sich auf ein Textelement, das implizit fokussiert ist und im Nahverweis vorkommt. ${ }^{18}$

Beispiel aus einer Rotpunkt-Nachricht:

Allerdings meinen einige Kletterer, daß diese Tour eher mit $7 c+z u$ bewerten sei. Wie dem auch sei, Anna reagierte schnell und stellte am letzten Tag des Jahres 1992 ihr Kletterniveau erneut unter Beweis, indem sie auf Mallorca eine andere mit 8 a bewertete Route kletterte.

Die Argumentstelle, die beim Valenzträger reagieren nicht versprachlicht wurde, korreliert mit einem impliziten anaphorischen Textelement. Es ergibt sich aus dem vorangehenden Satz (Anna reagierte "auf diese Behauptung").

Beispiel aus der Grif-Nachricht:

Guido Kostermexer je preplezal novo smer Shan-gri-la, jo ocenil z 8c, pravi pa, da bi znala biti celo 8c+. Izkazal se je tudi Avstrijec Manfred Stuffer, ki je avgusta preplezal smer Trick\&Track (8c), poleg te pa v plezališčih po Avstriji še veliko smeri z ocenami $8 b$ in $8 b+$.

Das Verb izkazati se ${ }^{19}$ (dt. sich hervortun) ist zweiwertig, ausgelassen wurde die fakultative Präpositionalphrase. Die leere Argumentstelle ist mittels eines implizit fokussierten Textelements aus dem Vortext zu aktualisieren. Dies erfolgt durch die Inferenzziehung des Lesers, der durch die Lexeme preplezati, smer die leere Position entsprechend ergänzen kann.

Im deutschen Sprachmaterial kommt die Argumentellipse in diesem Kontexttyp häufiger (30\%) vor als im slowenischen $(21 \%)$. Auch dieser Kontexttyp unterstiitzt die Referenzkontinuität und sorgt für eine kohärente Satzabfolge. Da das Argument nicht syntaktifiziert wird, motiviert die leere Position den Leser, eine intensive Lesart vorzunehmen und mitzudenken. Durch Ausfüllen von Lücken mit den aus der Textwelt gelieferten Informationen wird bei der Rezeption sozusagen eine innere Spannung hervorgerufen, wodurch der Leser unterhalten wird.

18 Nach DENTLER (1990) geht es um den Kotnexttyp B1.

19 Vgl. SSKJ, 2. knjiga, 1985, 109: izkazati se: 1. pokazati veliko sposobnost, pripravljenost, požrtvovalnost: učenci so se izkazali na tekmovanju; ekspr. izkazali so se in mi naredili obleko $\mathrm{v}$ dveh dneh; odlikovani so bili rudarji, ki so se izkazali ob nesreči; pri tem delu se nismo izkazali; naši nogometaši so se v zadnji tekmi (zelo) izkazali. Aus den angeführten Beispielen im Wörterbuch der slowenischen Schriftsprache geht hervor, daß dieses Verb zweiwertig ist. 
c) Die leere Stelle bezieht sich auf ein Textelement, das nicht als ein konkretes explizit oder implizit fokussiertes Textelement vorhanden ist, sondern Teil des Kommunikationsbereiches ist, vor dessen Hintergrund kommuniziert wird.

Beispiel aus der Rotpunkt-Nachricht:

Die Tour selbst ist mehr als 50 Meter lang. Sie beginnt in einer überhängenden Rinne, führt dann in ein 40 Grad steiles und 25 Meter ausladendes Dach und dann in eine Platte, die sehr schlechte Sicherungsmöglichkeiten bietet.

Beispiel aus der Grif-Nachricht:

$\mathrm{Ta}$, eden zelo redkih slovenskih lednih slapov na prisojni strani, je visok kakih sto metrov in ponuja dva raztežaja napete in lepe plezarije v zelo veliki naklonini.

Die nicht versprachlichte semantische Rolle des Rezipienten wäre beim Verb bieten, sl. ponujati mit einem Textelement wie "jemand, der bergsteigt/der klettert" bzw. "der Bergsteiger/Kletterer" (ähnlich auch für das Slowenische "plezalec", "alpinist") zu ergänzen, da die Proposition der Beispielsätze vor dem Hintergrund des spezifischen Kommunikationsbereiches ("Bergsteigerwelt") rezipierbar ist. Die leere Argumentstelle ist demnach mit einer Nominalphrase als Gattungsbezeichung zu besetzen. Wegen des Referenzbezugs auf den Kommunikationsbereich ist die Argumentstelle teilweise definit. Solche Argumentellipsen sind im slowenischen (10 $\%$ ) und im deutschen (16\%) Korpus vertreten. Gründe für die Argumentellipse liegen oft in der Sprachökonomie.

d) Die leere Stelle bezieht sich auf ein Textelement, das als Personalpronomen ich oder wir zu realiseren ist und das referenzidentisch mit dem Sender ist. Beispiel aus der Rotpunkt-Nachricht:

Kein Name, kein Anhaltspunkt, kein Garnichts! Lieber anonymer Chronist, bitte melde dich, sonst gehen wir zu Jörg Wontorra!

Im deutschen Beispiel ist die fakultative Präpositionalphrase ausgelassen, die leere Position ist senderbezogen. Sie wäre mit der fakultativen Präpositionalphrase bei uns zu verbinden, deren Personalpronomen referenzidentisch mit dem Sender ist.

Beispiel aus der Grif-Nachricht:

\section{Zlagali smo se oziroma so nas zavedli.}

Beim Verb zlagati se ist die thematische Rolle des Rezipienten in Form von einer fakultativen Dativergänzung ausgelassen worden, die leere Stelle wäre realisierbar mit dem Personalpronomen 2. Person Plural vam. Offenbar geht es um einen definiten Referenzbezug; aus dem sprachlichen und dem situativen Kontext läßt sich schlußfolgern, daß die Stelle empfängerbezogen ist.

Die Tatsache, daß in der Rotpunkt- und Grif-Nachricht die Relation Sender-Empfänger durch die Argumentellipse hervortritt, ist situationsbedingt. Durch solche Abweichungen entsteht ein lockerer Stil, der in dieser sozialen Situation erwartet wird. 


\section{ZUSAMMENFASSUNG}

Wie gezeigt wurde, haben die Argumentellipsen in deutschen und slowenischen weichen Nachrichten ähnliche Funktionen. Die Unterschiede betreffen vor allem die Auslassung obligatorischer Ergänzungen. Die Analyse hat jedoch gezeigt, da $\beta$ in beiden Korpora die kontexteingebettete definite Argumentellipse die Referenzkontinuität in einzelnen Textsequenzen stützt. Damit wird eine kohärente Lesart erzielt, denn der Leser wird durch die leere Argumentstelle motiviert, im Kontext nach der Vor- oder Nachinformation zu suchen, um die leere Argumentstelle entsprechend rezipieren zu können. Einige Beispiele können sogar nur mithilfe des Szenariowissens interpretiert werden, das der Leser dieser Zeitschrift besitzt und das der Sendererwartung nach - er aktivieren wird.

Umgangssprachlich markierte Argumentellipsen und verschiedene Arten des Referenzbezugs tragen zur lockeren Sprachgestaltung der weichen Nachricht bei. Die Wahl der Argumentellipse folgt demnach der Intention, eine von den Lesern erwartete Lockerheit in der weichen Nachricht zu erzielen.

\section{QUELLENVERZEICHNIS}

Nachrichten aus "Rotpunkt": 2/91: "Gefährlich, teuer und sieht gut aus", "Meeting", "Elbsandstein", "Exotik", "Vertical - Claude", "Vertical - Reunion", "Vertical - Weltcupsieger", "Vertical - Neuheit im Eis"; 2/92: "Jump"; 2/93: "Tough Stuffer", "Vollkommen unterschätzt", "Anna Ibanez"; 94: "Big Wall", "Dani 'Veloz' Andrada", "Retrobolting"; 4/94: "Eviva", "Altmühltal", "Action", 56 Bühler, "X=trem", "Stilfrage"; 5/94: "Hallenhalma", "Held der Arbeit", "Wie du mir, so ich dir", "Feiertage"; 6/94: "Alpen glühen", "Legenden", "Entspannte Atmosphäre", "Neues aus Plastikland", "Jailhouse Rock", "Southern Rock", "Schindlers Liste 2", "Kurz\&bündig", "Hoch lebe die Pfalz", "Die weiße Rose"; 1/95: "Keine 24 Stunden", "Misstress an Master's Edge", "Angie", "Clips", "Lost in..."; 2/95: "I found it allright", "Klettern ist die Hölle", "Klettern an der Nordseeküste", "Transzendental", "Berlin"; 3/95: "Von starken Jungen und verschenkten Frauen", "Über kurz und lang; 4/95: "Maximum am Minimum", "Roch 'n Road"; 5/95: "Jerry hebt ab", "Was uns spanisch vorkommt", "Handmade", "Wild Thing", "Bitte warten"; 6/95: "Was lange währt", "Speed", "Ramayana", "Schwindelnde "Höhen", "Späte Rache", "En Bloc", "Sperrungen in El Chorro", "Outdoor"; 1/96: "Devided Years", "Expeditionen"; 3/96: Nachrichten "Radja 8B+", "Robert Jasper", "Mönchs Eiszeit"; 4/96: "Fürstliche Leistung", "In eigener Sache"; 5/96: "Im Meer der Alpträume"

Nachrichten aus "Grif": 1/1: "Nove smeri v Mišji peči", "Janez Jeglič preplezal Mrtvaški ples", "Mirko Lebar proti Piscu", "Novo plezališče pri Velenju"; 1/2: "Fri, fri", "Erbestieldova zadnja na mastru v Arcu"; 1/3: "Slovenci uspešni na graškem festivalu", "Ponovljena Action direct (II)", "Alternativni programi na Šiji", "(Bodoče) Plezališče v bližini Bovca"; 2/4: "Preplezan nov slap", "Tevtonci", "Nagrada za film 
Namaste, Anapurna"; 2/5: "Francozi spoznavajo svet in domovino", "Tekmovanje v Zagrebu", "Aljaška divjina"; 2/6: "Deskanje z 8000", "Morje sanj"; 2/7: "Novice na tržaški način", "Jadralsko-plezalska odprava", "Sova v Ospu in na Gorenjskem"; 2/8: "Everest express", "Krisztof v Pakistanu", "7x5.14", "Gorski tek na Grintovec", "Spoznavaj svet in domovino", "Perkov seznam", "Usodno neurje v Nepalu", "Plezanje v kamnitem gozdu"; 2/9: "Črna burja", "Žur v Špornu", 97/10: "Janez Jeglič tokrat prvič prvi, prvi", "Norvežani - polarni razskovalci št. 1", "Od Španije do Galaktike".

\section{LITERATURVERZEICHNIS}

BRAČIČ Stojan (1994): Die Valenz als textgrammatische Kategorie. Linguistica, Ljubljana, Slovenija, 34:1, S. 47-55.

DeBEAUGRANDE Robert-Alain, DRESSLER Wolfgang Ulrich (1981): Einführung in die Textlinguistik. Tübingen.

DENTLER Sigrid (1990): Verb und Ellipse im heutigen Deutsch. Zum "Fehlen" verbabhängiger Bestimmungen in Theorie und Praxis. Göteborger germanistische Forschungen 31. Goterna, Kungälv.

ENGEL Ulrich, SCHUMACHER Helmut (1976): Kleines Valenzlexikon deutscher Verben. Tübingen.

GERLING M., ORTHEN N. (1979): Deutsche Zustands- und Bewegungsverben (=Studien zur deutschen Grammatik 11), Tübingen.

HEINEMANN Wolfgang, VIEHWEGER D. (1991): Textlinguistik. Eine Einführung. Tübingen.

HELBIG Gerhard (1992): Probleme der Valenz- und Kasustheorie. (=Konzepte der Sprach- und Literaturwissenschaft 51). Tübingen.

HELBIG Gerhard, SCHENKEL Wolfgang (1991): Wörterbuch zur Valenz und Distribution deutscher Verben, 8., durchgesehene Auflage. Tübingen.

HERINGER Hans Jürgen (1984): Neues von der Verbszene. In: Pragmatik in der Grammatik. Hg. Gerhard Stickel. Düsseldorf.

HEUSINGER Siegfried (1995a): Pragmalinguistik. Ein Lehr- und Übungsbuch. Frankfurt am Main.

HEUSINGER Siegfried (1995b): Textsorten in der interkulturellen Kommunikation ein Problemaufriß. in: Prototypisches in Textsorten, Sammelband zu den Beiträgen des internationalen Symposions. Hg. J. Orešnik, S. Heusinger et al. Linguistica XXX, 1, Ljubljana, S. 7-20.

KREVS Uršula (1996): Argumentellipse in schriftlicher Kommunikation unter der Bedingung einer speziellen sozialen Situation. Magisterarbeit. Ljubljana.

LÜGER Heinz-Helmut (1995): Pressesprache. Tübingen.

OREŠNIK Janez (1992): Udeleženske vloge v slovenščini. Ljubljana.

SHOPEN Timothy Ames (1972): A Generative Theory of Ellipsis. (Diss.), University of California, Los Angeles.

SLOVAR SLOVENSKEGA KNJIŽNEGA JEZIKA I-V (1970, 1975, 1979, 1985, 1991). Ljubljana. 
TOPORIŠIČ Jože (1984): Slovenska slovnica. Maribor.

WUNDERLICH Dieter (1985): Über die Argumente des Verbs. In: Linguistische Berichte 97, S. 183-227.

\author{
Povzetek \\ ELIPSA ARGUMENTA V "MEHKI" VESTI V NEMŠČINI IN V SLOVENŠČINI
}

Elipsa argumenta je $\mathrm{v}$ članku prikazana primerjalno s skladenjskega in besediloslovnega vidika. Besedilno vpetost elipse argumenta, tj. izpusta dopolnila, je mogoče opredeliti glede na določnost oz. nedoločnost nezasedenega mesta. Ta lastnost elipse argumenta razkriva referenco mesta izpusta in tako kaže na povezavo eliptǐne skladenjske strukture $z$ besedilom. Pozornost raziskave je bila nadalje usmerjena $v$ besedilno vrsto "mehka" vest, v kateri želi pisec besedila poleg posredovanja informacij usmeriti bralčevo pozornost tudi na jezikovno oblikovanost besedila in ga $\mathrm{z}$ retoričnimi figurami, jezikovnimi igrami ipd. zabavati. Analiza primerov elips argumenta iz "mehkih" vesti v nemški in slovenski športni reviji je pokazala, da se prazna mesta navezujejo na razlið̌ne elemente $v$ jezikovnem in situacijskem sobesedilu. Takšne določne elipse arugumenta so lahko učinkovito sredstvo za tvorbo koherence besedila. Hkrati pa prispevajo izpusti obveznih in neobveznih dopolnil $\mathbf{k}$ sproščeni oblikovanosti besedila, ki je v besedilni vrsti "mehka" vest pričakovana. 